\title{
Classifying Economic Areas for Urban Planning using Deep Learning and Satellite Imagery in East Africa
}

\author{
DAVY UWIZERA ${ }^{1,2}$, (Member, IEEE), CHARLES RURANGA ${ }^{1}$, AND PATRICK MCSHARRY. ${ }^{1,2,3}$, \\ (Member, IEEE) \\ ${ }^{1}$ African Center of Excellence in Data Science (ACE-DS), University of Rwanda, Rwanda \\ ${ }^{2}$ Carnegie Mellon University Africa, Kigali, Rwanda \\ ${ }^{3}$ Oxford MAN Institute of Quantitative Finance, University of Oxford, UK \\ Corresponding author: Davy Uwizera.
}

\begin{abstract}
Monitoring and assessing the distribution of economic areas in East Africa such as low and high income neighborhoods, has typically relied on the use of structured data and traditional survey approaches for collecting information such as questionnaires, interviews and field visits. These types of surveys are slow, costly and prone to human error. With the digital revolution, a lot of unstructured data is generated daily that is likely to contain useful proxy data for many economic variables. In this research we focus on satellite imagery data with applications in East Africa. Recently East African cities have been developing at a fast pace by building new infrastructure and constructing innovative economic zones. Moreover with increased urban population, cities have been expanding in multiple directions affecting the overall distribution of areas with economic activity. Automatic detection and classification of these areas could be used to inform a number of policies such as land usage and could also assist with policy enforcement monitoring. On the other hand, the distribution of different economic areas in a specific city could provide proxies for various economic development variables such as income distribution and poverty metrics. In this research, we apply deep learning techniques to satellite imagery to classify and assess the distribution of various economic areas of a specific region for urban planning. By benchmarking performance against traditional machine learning models, results show that deep learning techniques yielded superior performance. ResNet50 outperforms deep models considered and achieves a classification accuracy of $98 \%$.
\end{abstract}

INDEX TERMS classification, deep learning; satellite imagery; transfer learning; urban planning; remote sensing; monitoring.

\section{INTRODUCTION}

$\mathbf{E}$ CONOMIC areas range from low and high income neighborhoods, industrial zones, trade areas, schools etc [50] [53] [28]. There are various geographical factors and economic drivers which influence the distribution of economic areas including government policies. The spatial distribution of various economic areas has been used in various urban economic planning [29] such as transport [38], city expansion [36] and land usage policies [54]. Moreover, their distribution influences daily individual and household economic decisions depending on their corresponding geographical location [34], hence, indirectly influencing a number of macro-economic variables. The analysis and monitoring of the spatial distribution of these areas in East
Africa has previously relied on the use of structured data and traditional approaches for data collection such as paper questionnaires, face-to-face interviews and field visits [1]. These survey methods are prone to human error and are often slow and costly [11]. Yet, with the digital revolution a lot of unstructured data is generated daily that is likely to contain useful proxy data in line with various economic variables, and could be analyzed to support existing policy and generate new insights [10]. The sources of data range from daily captured images by individuals, government, social media as well as satellite imagery, which is our target data source in this research.

There are different types of satellite imagery data to consider such as night-time lights [40], weather estimates [16], 
vegetation indices [57] and infrastructure [42]. There are various patterns and relationships that have been identified from big data sources which can be utilised to address various challenges, resulting in different use cases. This includes, but is not limited to, transport demand prediction [41], conflict outbreak monitoring [31], disease outbreak prediction and spatial area labelling [32].

Spatial area labelling and access to digital maps have recently inspired innovations in technology, and contributed various use cases such as self driving cars [63] and automatic optimal routing [33]. Currently digital map labeling could act as the ground truth for validation assessment or provide a secondary data source for various research studies in line with spatial areas such as economic area classification, which we will focus on in this study.

Using deep learning and transfer learning [56], our research seeks to classify spatial economic areas under five categories (residential high income, residential middle income, residential low income, commercial buildings and industrial areas) in East Africa, by focusing on Rwanda. East African countries have promoted infrastructure development with various programs initiated to facilitate city infrastructure expansion. Some of the initiatives included dispatching big government institutions in various locations of city outskirts rather than having them condensed in one central place of the city [2]. Moreover there has been enormous effort to renovate cities with new master-plans to promote more infrastructure and greenness. However, reaching sustainable urban development has been a challenge in developing countries [13].

With rapid urban migration in the region, under the absence of a stable economic base, there has been an increasing trend of overpopulation in urban areas, resulting in inactive rural areas with shifting demographics, decreased tax base and slum formulations in the city [35]. Slums affect the expansion of urban infrastructure in various ways. Normally they provide homes for many families on a very small area of land. Hence in most cases, the government has to expropriate or find ways to relocate various families that would be affected in order to expand the infrastructure in the area. Hence, the ability to analyze and classify spatial economic areas will have various applications in facilitating monitoring of country infrastructure trends such as slum formulation, distribution of different types of residential areas and could guide various policies in urban planning.

As stated previously, urban economic monitoring has been relying on traditional paper surveys and questionnaire methods in most developing countries. Although this has been helpful for various case studies, there are a number of downsides as we highlighted such as timeliness, high cost, human error and enumerator fraud. By using artificial intelligence techniques such as machine learning, it is possible to utilise satellite imagery and in parallel a number of these issues are automatically mitigated.

While machine learning techniques and deep learning methods in particular are now viewed as state-of-art in various fields, their application on unstructured proxy data such as imagery for economic activities monitoring is still limited in developing countries [58]. Consequently, some of the machine learning techniques such as deep-learning, which have demonstrated great potential for the analysis and modeling of unstructured data, are not widely used in economic studies. This research also seeks to fill this gap by contributing to existing academic knowledge of applying machine-learning techniques to solve real life problems in sub-saharan Africa using deep learning [19] [62] and transfer learning [43] [51].

\section{A. LITTERATURE REVIEW}

Economic area analysis and classification have been of great interest to various researchers especially for economic monitoring and policy making [45]. Patterns underlying the formation of economic areas and the resulting differences between associated economic classes have been used in addressing a multitude of research problems across the globe in various sectors.

Czapiewski et al. assessed the impact of location on the role of small towns in regional development in poland [14]. The researchers demonstrate how depending on the location of the small towns, they can play various roles such as connecting rural and urban areas and serving as a local engine for economic development. By classifying eighty six target towns in three categories, small, medium-sized and big and using a two step methodology [39], it was found that the location of small towns has a significant impact on boosting various economic factors such as education, access to health care and cultural activities [14].

Location is one of the important features in spatial area classification and has been used within other case studies. Lamnisos et al. analyzed the correlation between geodemographic areas and mortality. Using a Poisson distribution for mortality estimation and K-means for area classification the researchers found, at $95 \%$ confidence interval, that rural areas and semi-rural areas had $44 \%$ and $36 \%$ higher premature mortality rate respectively compared to metropolitan areas[27]. Further details of K-means and Poisson log-linear models can be found in [24] [17]. Bański et al. conducted research on the classification of rural areas for land usage policy in Poland. By investigating economic structure, transport-wise accessibility and development dynamics, the authors estimated the state of development of rural areas [3].

Spatial distribution and classification of economic areas is a broad subject with great relevance for the monitoring of economic activities. Barbero et al assessed the correlation between wages and the spatial distribution of various industries in general equilibrium trade models [4] for asymmetric markets [7]. Li et al [30] highlighted the geographical location determinants of high-growth firms by assessing factors associated with their emergence. Basu et al, analyzed house prices and spatial autocorrelation [6]. Using estimated generalized least squares [7] their research results showed that there is statistically significant spatial autocorrelation for house transaction prices. Yoo et al, also analyzed the spatial autocorrelation and spatial heterogeneity of house 
prices controlled by the presence and absence of agricultural conservation easement policy [64]. Some researchers have also studied the relationship between spatial distribution of neighborhoods and violent crimes such as Barton et al [5], Papachristos et al [44], He et al [20] hence demonstrating that violent crimes influence various economic activities in corresponding locations.

While the location of economic areas has an impact on economic activities there are also factors influencing the formation of economic areas. Hodgson highlighted some of these factors such as transport infrastructure [23]. The paper shows that there was a significant effect of railroad construction on the geographical distribution of towns in the Western states of the USA. Clausing et al measured the impact of taxes and big firms headquarters locations [12]. Other factors affecting the distribution of economic areas include but are not limited to rural-urban migration[47], natural disasters[15] and weather conditions [48].

There are various ways of analyzing and classifying economic areas that aim to improve upon the traditional use of surveys. Kussul et al used deep learning, specifically convolution neural networks $(\mathrm{CNN})$, to classify land cover and crop types from satellite imagery [26]. The results of their research showed that deep learning outperformed random-forest with a significant margin. Sidike et al applied deep learning on satellite imagery to label diverse agricultural landscapes [52] for field management. The paper expresses how remote sensing technologies are cost-effective compared to traditional mapping practices with intensive field surveys and proposes a deep learning method for mapping 19 different agricultural landscapes and compared the accuracy against support vector machine and random-forest. Deep learning scored the highest accuracy with $86 \%$.

Oshri et al used deep learning and satellite imagery to assess infrastructure quality in Africa [42]. While infrastructure quality is one of the important features in United Nations Sustainable Development Goals, the research highlights how monitoring progress in developing regions is still expensive using traditional survey methods and proposed a remote sensing approach using deep learning. By training convolutional neural networks researchers were able to predict the ground truth labels of the Afrobarometer round five survey. The labels comprised Electricity, Sewerage, Piped Walter and Roads. The researchers achieved classification accuracies of $88 \%, 86 \%, 74 \%$ and $78 \%$ respectively.

Berriel et al used deep learning for automatic classification of crosswalks [8]. The research focuses on filling the gap of zebra crossing classification problem for automatic retrieval and annotation of crosswalks satellite imagery data. The results of the research showed that deep learning could be used to perform crosswalk classification with a $97 \%$ accuracy on a global scale.

Satellite imagery and deep learning have also been used in surface object segmentation, showing the ability of deep learning models to not only classify, but also understand the boundaries of the object pattern in the learning pro- cess. Bishcke et al segmented building footprints using deep learning and satellite imagery [9]. The research focused on improving semantic segmentation approaches with a multitask loss. The results showed they were able to increase accuracy by a significant eight percent on existing state-ofart methods in semantic segmentation.

Khan et al conducted research on automatic target object detection in satellite images [60]. The authors highlight how traditional image feature extraction such as Histogram of Oriented Gradients, Gabor Feature and Hough Transformation don't work well for huge data of varying size, orientation and noisy background. They propose an edge boxes method using a convolution neural network to filter the set of target object pixels position. Van also used a deep learning method called YOLT (you only look twice) for faster detection of small and big objects in satellite imagery such as vehicles, airplanes, boats, buildings and airports [25].

Clearly a lot of research has been done in line with the use of deep learning on satellite imagery and yielded tangible results over traditional image classification and detection methods. In summary, the combination of deep learning and satellite imagery has proved to be very cost-effective and less time consuming compared with traditional field intensive survey methods.

In this research we seek to use deep learning methods for classification of economic areas in East Africa. The five categories considered are (1) residential low-income; (2) residential middle-income; (3) residential high-income; (4) commercial buildings; and (5) industrial zone. In the following section we discuss our methodology starting with the exploration of our data.

\section{METHODOLOGY}

\section{A. DATA}

In this research we use data from a number of different sources of satellite imagery. Below we describe and visualize various metrics of the datasets being considered. Satellite imagery is retrieved from Google earth which is supported by Data SIO (Scripps Institution of Oceanography), NOAA (National Oceanic and Atmospheric Administration), US. Navy (United States Navy), NGA (National GeospatialIntelligence Agency), GEBCO (General Bathymetric Chart of the Oceans), Image Landsat, and Image IBCAO (International Bathymetric Chart of the Arctic Ocean).

Using random sampling of spatial area in Kigali per target area, 342,843 thousands images were retrieved under the five categories: residential high income (78941), residential low income(162501), residential middle income(101401), commercial building, (67400) and industrial zone,(24400). For the industrial zone, we also included some images from Nairobi, Kenya industrial spatial area. The average number of samples for a category is 86929 . The size of the sample per category is proportional to the size of the spatial target area considered per category. Kigali is located at latitude:1.985070 and longitude:-1.985070, coordinates. Nairobi is 
located at latitude:-1.286389 and longitude:36.817223, coordinates.

Our target areas were guided by field survey results obtained from Ernest et al [59] on spatial distribution of the affordable housing projects and informal settlements in Kigali city as highlighted in the map below. Figure 1 .

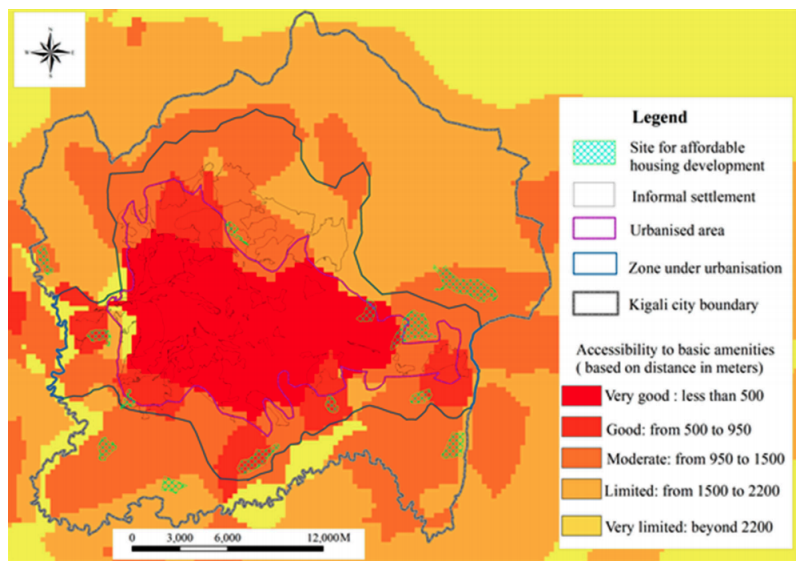

FIGURE 1. Source: Ernest et al [59], Spatial distribution of the affordable housing projects and informal settlements in Kigali city.

Using human review and target areas in Figure 1, images which were labeled as low incomes, Figure 2, were mostly slums without easy access to main roads, minimal or no neighborhood road infrastructure and primarily consisted of small compounds.

Labels for middle income areas were given to images which consisted of bigger compounds and evidence of road access. Furthermore there were a few houses which share some of the characteristics of the houses that were found in the low residential income category, Figure 3.

Images which were labeled under residential high income had easy access to roads, bigger compounds and had modern roofs overall from the space view. Figure 4

Images containing a collection of skyscrapers and other low elevation buildings, mainly in the central business district and towns were labeled as commercial buildings. Figure 5

Images labeled as industrial zone category are typically made up of large flat buildings. Most of these images were retrieved from known industrial zones locations in East Africa focusing on Kigali and Nairobi.

\section{B. METHODS}

Deep learning methods are used to classify economic areas under five distinct categories: residential low income, residential middle income, residential high income, commercial buildings and industrial areas. We select three deep neural network architectures: Mobilenet version 2 (MobilenetV2), Inception version 3 (Inceptionv3), Resnet50 version 2 (Resnet50V2) for training. We also adopt deep transfer learning [56] with imagenet pre-trained weights.

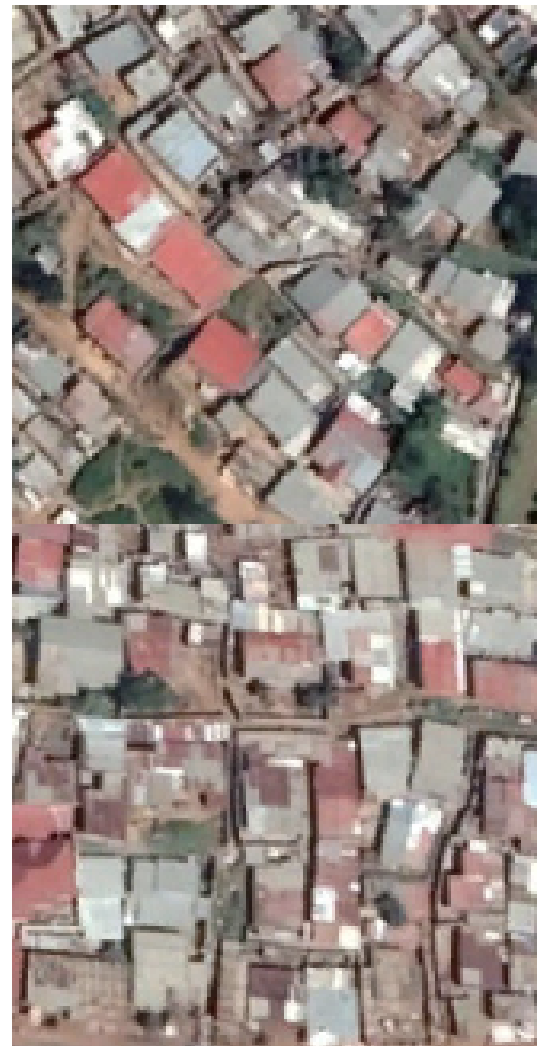

FIGURE 2. Residential low income sample images

\section{1) MobileNetV2}

Mobilenet version is based on inverted residual structure with depth-wise separable convolutions. It minimizes nonlinearity in narrow layers to improve robustness in representational power. The model is light-weight, but has proved to maximize both accuracy and computational speed in various computer vision tasks. MobileNetV2 architecture could be found in Sandler et al [49].

\section{2) InceptionV3}

By using factorized convolutional layers and aggressive regularization, the model architecture seeks to improve accuracy and at the same time minimize added computation per layer. InceptionV3 architecture is implemented as in Szegedy et al [55].

\section{3) ResNet50V2}

Resenet50V2 is among deep residual network architectures, they focus on learning residual functions rather than learning unreferenced functions. Resnet architectures demonstrated higher accuracy and fast convergence[60]. Resnets models fall in different categories depending on the number of layers used in model building. The most common are Resnet18, Resnet34, Resnet50, Resnet101 and Resnet153 [21]. In this research we use Resnet50V2 [22]. Resnets add non-linearity between layers and the main difference between Resnet ver- 


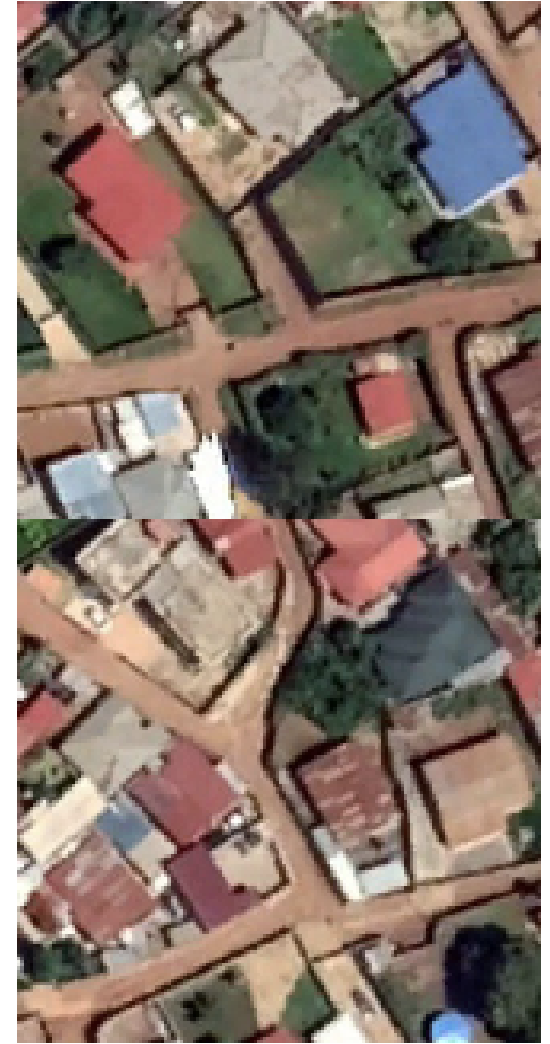

FIGURE 3. Residential middle income sample images

sion 1 and Resnet version 2 is the removal of non-linearity in the last layer on version 2. This was done to promote easy continuous connection in a form of identity connections. Resnet architecture used are presented in $\mathrm{He}$ et al [22].

As previously mentioned, MobileNetV2, Resnet50V2 and InceptionV3 are used as our base-models for our research task. The models are initiated without the final layer (head) and imagenet weights are used with these models to speed up learning and minimize risk of convergence to local minimum. We add one dense layer of size 512 with sigmoid activation and the output layer with softmax activation.

\section{4) Machine learning models using manual feature extraction}

We combine color distribution with sobel filtering techniques to detect edges, angles between edges using vertical and horizontal gradients [18], and use local binary pattern [46] and histogram of gradient orientation for feature extraction of our benchmark models. Benchmark models considered are Random Forest (RF), Support Vector Machine (SVM) and Knearest Neighbors (KNN).

\section{5) Visualization}

To visually understand the separability and compactness of considered predictive models, we use t-stochastic neighborhood embeddings (t-SNE) [37]. t-SNE converts similarities between data points to joint probabilities.

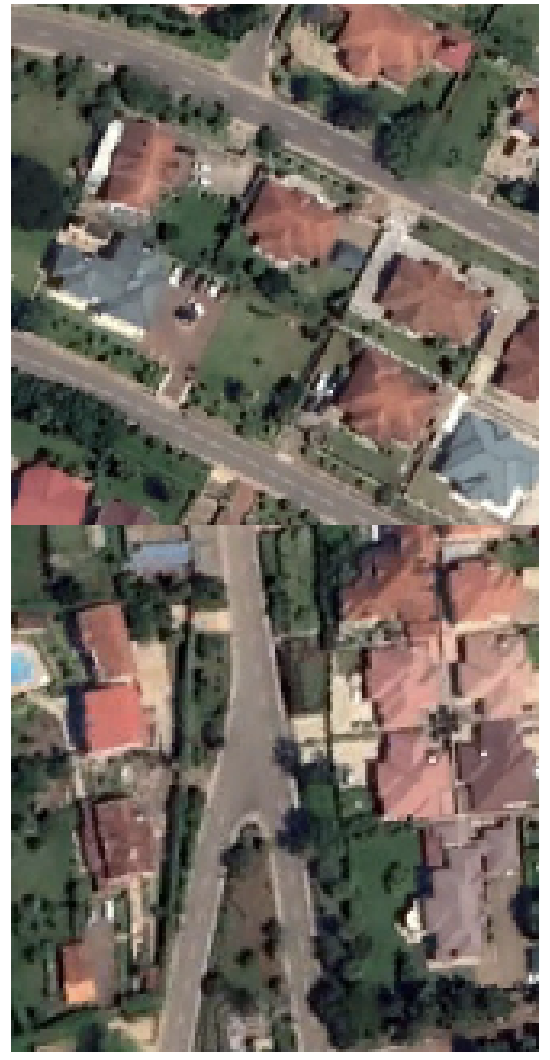

FIGURE 4. Residential high income sample images

\section{TESTING}

RF, SVM and KNN models are used on features extracted discussed in section II-B4. The dataset, as described in II-A section, is divided into training and testing dataset. $70 \%$ is used for training and $30 \%$ is used for testing on both our target models and benchmark models. The test results are used to evaluate both accuracy and speed in order to draw conclusions about the best model to recommend for policymakers and practitioners.

\section{RESULTS \& DISCUSSION}

\section{A. DATA ANALYSIS}

While shape and size is a clear distinct pattern between economic areas as shown in Figures-4,3,2,5,6, color crossareas pattern could be better presented with a histogram analysis.

Although top roof and aerial view color components may not as a pattern apply much in developed countries, it turns out to be very crucial in developing countries, particularly in the area of East Africa. This is due to the fact that high income areas roof aerial view and roads tend to be neat with paved roads compared to middle income and low income areas. The same applies for commercial building areas versus industrial zones.

Using random sampling of 5000 images per category, below we present the color histogram distribution of our 


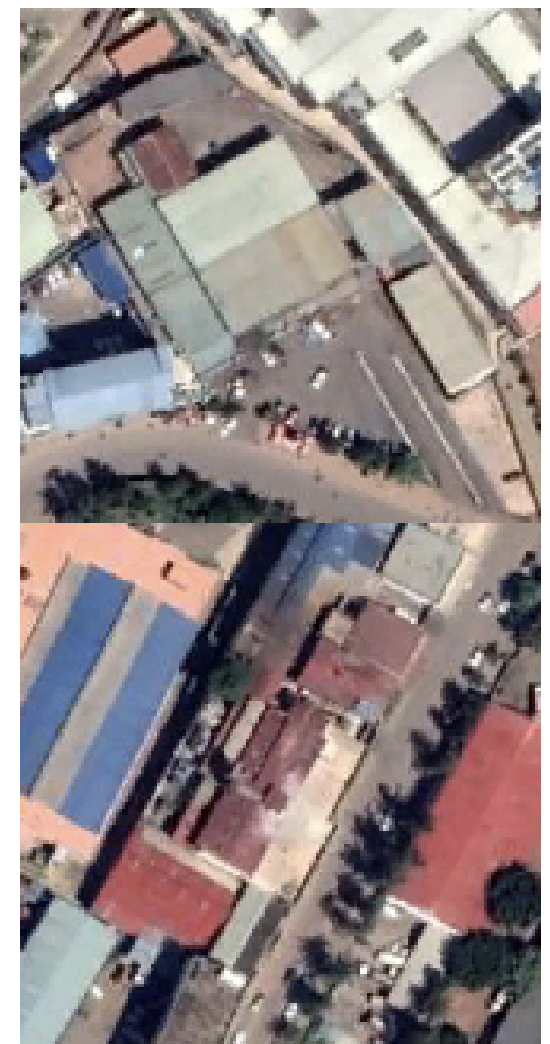

FIGURE 5. Commercial building sample images Images

dataset.

\section{1) Color Histogram Analysis}

In Figures-7,8,9, we plot color histograms by channels; Red, Blue, Green (RGB) and present further color patterns behind our economic areas.

Figure-7 shows that residential low-income category blue channel spikes in between fifty and ninety pixel values, and one hundred and sixty and one hundred and eighty compared to green and red channels which are skewed to the right with spikes in between seventy and one hundred and ten pixels values. Overall, this shows that the tonality of residential lowincome tends toward zero.

On the middle income side, Figure- 8 , the blue channel spikes are between thirty and fifty and one hundred on the left side and seventy and one hundred and eighty on the right side with a more narrow curve angle turning area compared to the low-income category. Green and red channels of the category are also left-skewed but with more variation compared to low-income category. It shows that the middle-income residential category has more color variation compared to lowincome residential category.

This is likely due to the fact that designs in middle class residential are more variant. In most of the cases middle class neighborhoods are made of houses which have been gradually improved from low to middle class houses rather

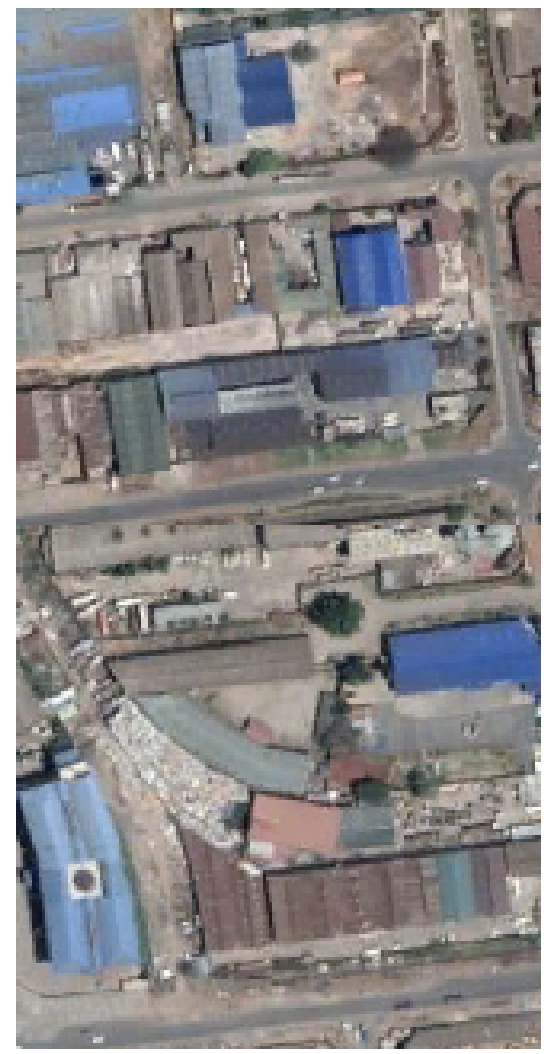

FIGURE 6. Industrial zone sample images
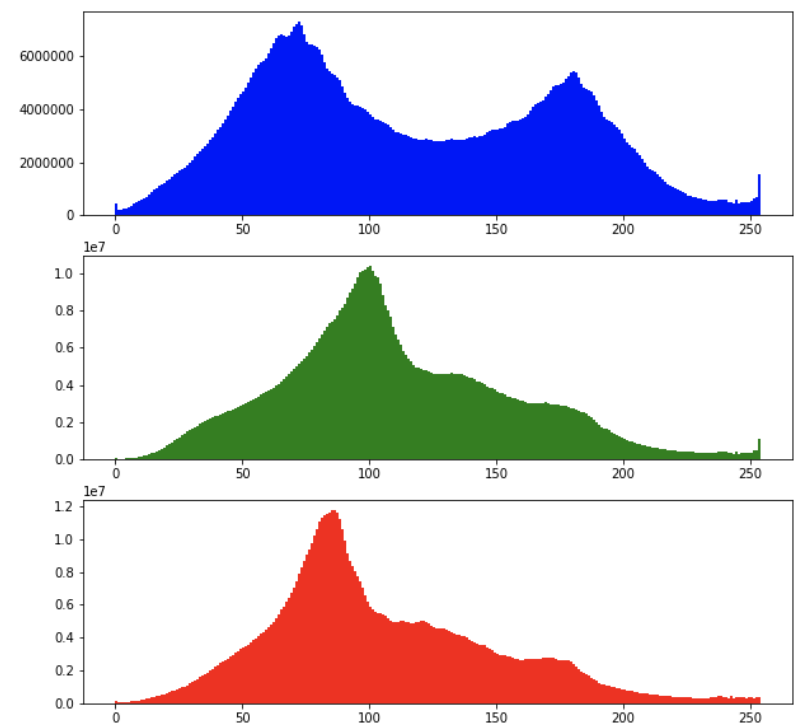

FIGURE 7. RGB color histogram for residential low income

than newly built houses from scratch. Hence, making their designs and roof colors to be more variant compared to low income class which tend to use the same form of cheap house materials.

Looking at residential high-income, Figure-9 shows that 

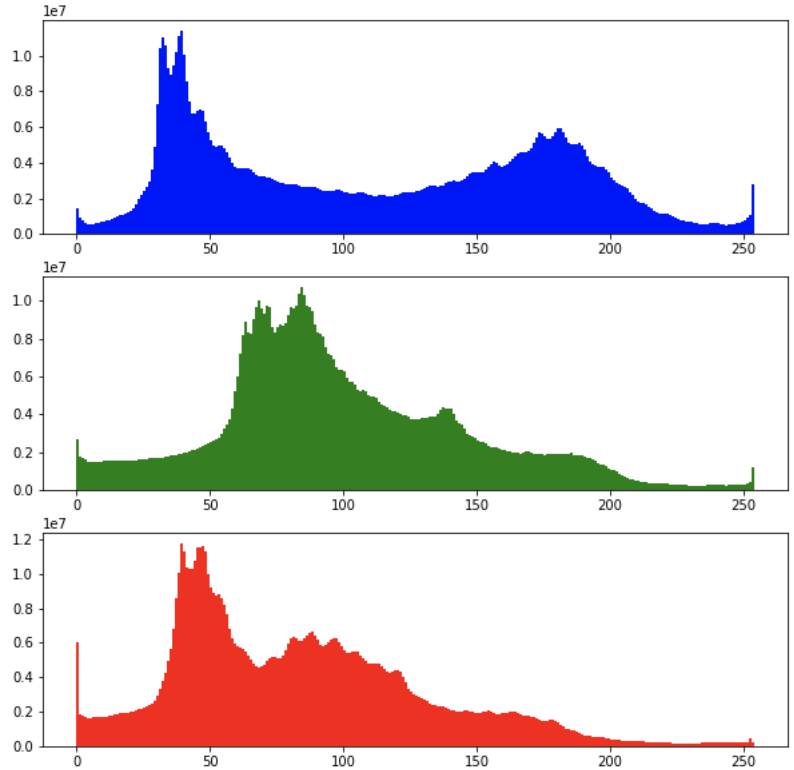

FIGURE 8. RGB color histogram for residential middle income

histogram values for all channels tend to spike all together in central with few spikes and minimal spikes intervals. This implies that high income residential category color is normally distributed with less variation compared to both lowincome and high income categories. The reason behind this is often linked to building regulations and naturally formed oligopolistic house design contractors.
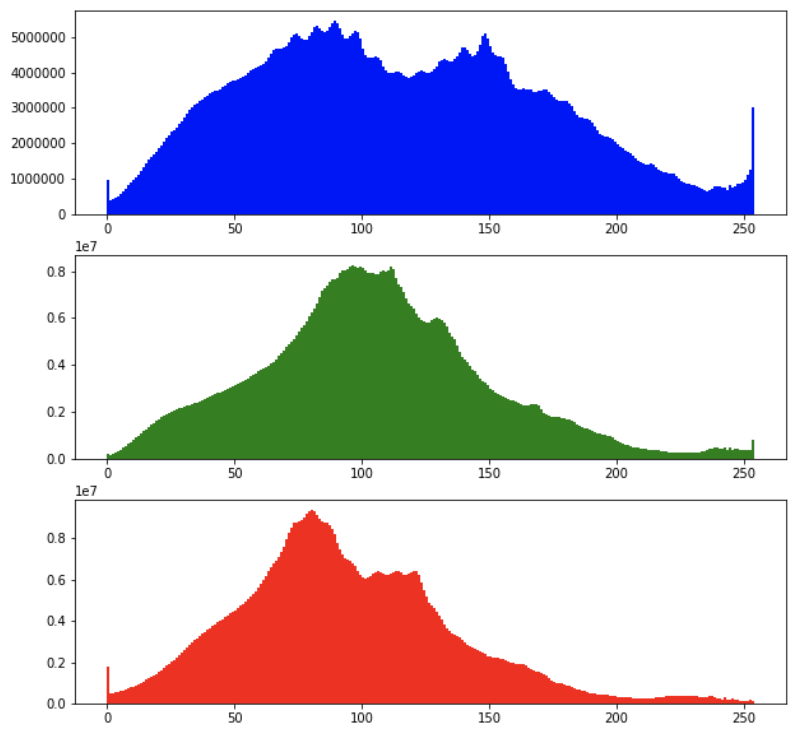

FIGURE 9. RGB color histogram for residential high income

\section{B. MODELING}

Each model was trained on batch 32, and 16 epochs with weight decay of 5e-04 and the overall accuracy of MobileNetV2, InceptionV3 and Resnet50 is $93 \%, 95 \%, 98 \%$ re- spectively. Input shape used was $224 \times 224$ for MobileNetV2 and Resnet50, and 299x299 for inception. In the following parts of this section we discuss in detail the classification results of our three target models.

\section{1) MobileNetV2}

Below we present detailed classification results of MobilenetV2, Table-1, and visualize the separability and compactness of the embedding layer output per predicted class using t-SNE, Figure-10.

TABLE 1. MobileNetV2 classification results

\begin{tabular}{|l|r|r|r|r|} 
& precision & recall & f1-score & Support \\
Commercial & 1 & 0.94 & 0.97 & 20220 \\
Industrial & 0.71 & 0.91 & 0.8 & 7320 \\
Residential_high & 1 & 0.95 & 0.97 & 23682 \\
Residential_mid & 0.83 & 0.91 & 0.87 & 30420 \\
Residential_low & 0.99 & 0.93 & 0.96 & 48750 \\
Accuracy & & & & 0.93
\end{tabular}

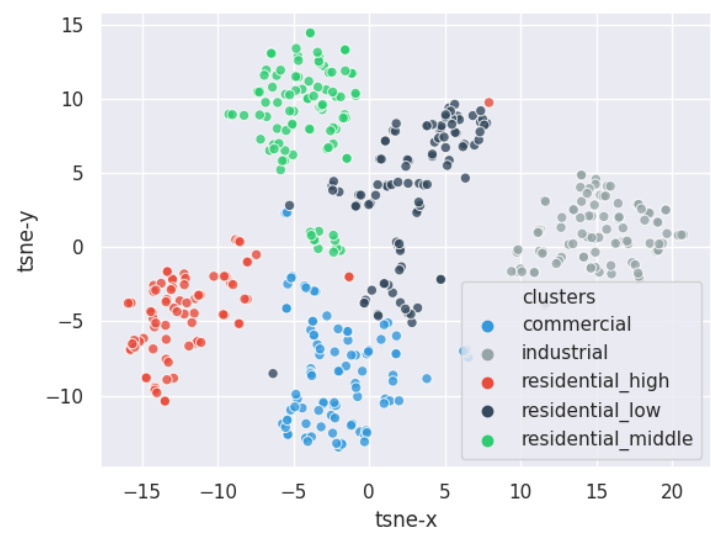

FIGURE 10. t-SNE visualization of MobileNetV2 embedding layer sample output, 80 images per category

The results in Table-1 show that MobileNetV2 scores 93\% overall accuracy on the five categories. Commercial and residential high are the most well classified categories by the model.

\section{2) InceptionV3}

Below we present detailed classification results of InceptionV3, Table-2, and visualize the separability and compactness of the embedding layer output per predicted class, Figure-11, the same way we did in the previous section.

Inception scores $95 \%$ overall accuracy on the five categories. Commercial, industrial and low residential areas are the most well classified categories by the model. Table- 2 .

\section{3) ResNet50V2}

Same as in the previous two sections, detailed classification results of Resnet50V2 are presented in this section, Table-3, 
TABLE 2. InceptionV3 classification results

\begin{tabular}{|l|r|r|r|r|} 
& precision & recall & f1-score & Support \\
Commercial & 1 & 0.96 & 0.98 & 20220 \\
Industrial & 1 & 0.93 & 0.96 & 7320 \\
Residential_high & 0.8 & 0.97 & 0.87 & 23682 \\
Residential_mid & 0.98 & 0.93 & 0.95 & 30420 \\
Residential_low & 1 & 0.95 & 0.97 & 48750 \\
Accuracy & & & & 0.95
\end{tabular}

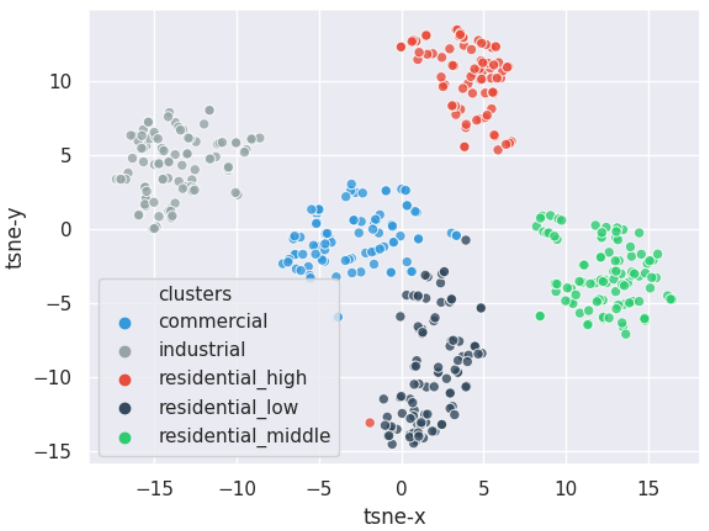

FIGURE 11. t-SNE visualization of InceptionV3 embedding layer sample output, 80 images per category

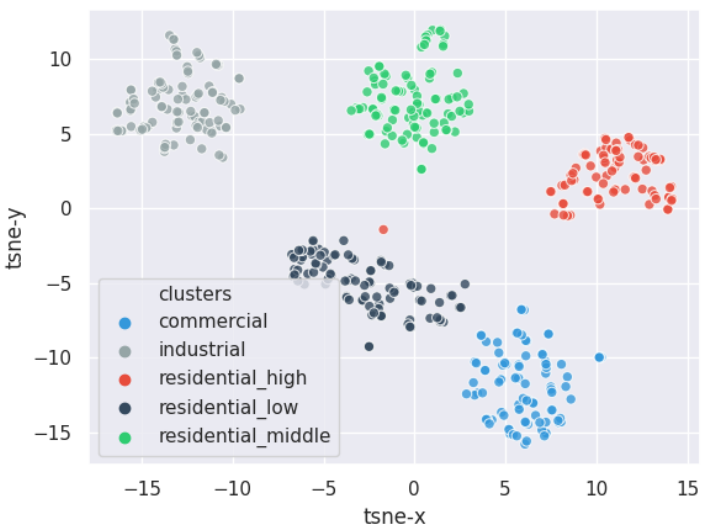

FIGURE 12. t-SNE visualization of ResNet50V2 embedding layer sample output, 80 images per category

with the visualization the separability and compactness of the embedding layer output per predicted classes, Figure-12.

TABLE 3. Resnet50V2 classification results

\begin{tabular}{|l|r|r|r|r|} 
& precision & recall & f1-score & Support \\
Commercial & 1 & 0.99 & 0.99 & 20220 \\
Industrial & 0.86 & 0.96 & 0.91 & 7320 \\
Residential_high & 0.99 & 1 & 0.99 & 23682 \\
Residential_mid & 1 & 0.96 & 0.98 & 30420 \\
Residential_low & 0.98 & 0.98 & 0.98 & 48750 \\
Accuracy & & & & 0.98
\end{tabular}

Table-3 shows that Resnet50V2 outperforms the rest of deep learning models by keeping a high accuracy across categories, especially for Commercial, Residential High and Residential Middle categories.

\section{4) Benchmark Models}

TABLE 4. SVM classification results

\begin{tabular}{|l|r|r|r|r|} 
& precision & recall & f1-score & Support \\
Commercial & 1.00 & 0.70 & 0.82 & 20220 \\
Industrial & 0.48 & 0.94 & 0.64 & 7320 \\
Residential_high & 0.95 & 0.76 & 0.85 & 23682 \\
Residential_mid & 0.75 & 0.60 & 0.67 & 30420 \\
Residential_low & 0.75 & 0.82 & 0.78 & 48750 \\
Accuracy & & & & 0.75
\end{tabular}

SVM scored the overall accuracy of $75 \%$ with highest accuracy results on residential high income areas. Table- 4 .

TABLE 5. RF classification results

\begin{tabular}{|l|r|r|r|r|} 
& precision & recall & f1-score & Support \\
Commercial & 1.00 & 0.74 & 0.85 & 20220 \\
Industrial & 0.56 & 0.88 & 0.68 & 7320 \\
Residential_high & 0.91 & 0.84 & 0.88 & 23682 \\
Residential_mid & 0.78 & 0.72 & 0.75 & 30420 \\
Residential_low & 0.78 & 0.82 & 0.80 & 48750 \\
Accuracy & & & & 0.79
\end{tabular}

RF scored the overall accuracy of $79 \%$ with highest accuracy results on residential high income areas. Table-5.

TABLE 6. KNN classification results

\begin{tabular}{|l|r|r|r|r|} 
& precision & recall & f1-score & Support \\
Commercial & 1.00 & 0.80 & 0.89 & 20220 \\
Industrial & 0.56 & 0.88 & 0.68 & 7320 \\
Residential_high & 0.92 & 0.86 & 0.89 & 23682 \\
Residential_mid & 0.83 & 0.70 & 0.76 & 30420 \\
Residential_low & 0.83 & 0.87 & 0.85 & 48750 \\
Accuracy & & & & 0.82
\end{tabular}

KNN scored the overall accuracy of $82 \%$ with highest accuracy results on commercial building areas. Table-6.

Table-7 highlights how each classifier considered compares for each economic area.

The results show that Resnet50V2 outperforms the rest of the models on commercial and all residential categories. However its performance falls under the InceptionV3's on industrial zone area classification.

The results also show that deep learning models far outperform traditional manual feature extraction machine learning based models.

\section{APPLICATION}

Using the best model highlighted in previous section, we showcase that the pattern learnt from the dataset in this research could be used from pixel to legend and map using Viola-Jones [61] sliding window. Hence, demonstrating that the model built could be applied for instant mapping or improvement of current survey based maps in urban planning, see Figure 13. 
TABLE 7. All models f1-score results per economic area

\begin{tabular}{l|rr|r|r|r|} 
& Commercial & Industrial & Residential high & Residential mid & Residential low \\
Resnet50V2 & 0.99 & 0.91 & 0.99 & 0.98 & 0.98 \\
InceptionV3 & 0.98 & 0.96 & 0.87 & 0.95 & 0.97 \\
MobilenetV2 & 0.97 & 0.8 & 0.97 & 0.87 & 0.96 \\
KNN & 0.89 & 0.68 & 0.89 & 0.76 & 0.85 \\
RF & 0.85 & 0.68 & 0.88 & 0.75 & 0.80 \\
SVM & 0.82 & 0.64 & 0.85 & 0.67 & 0.78
\end{tabular}

Figure 14 shows that you could use results obtained in this research to localize and map the spatial distribution of economic areas considered in this research with high accuracy. However, detection results show that in gray areas such as fields, pastures and roads, the model is likely to get them wrong if they are at the edge of the image view. Hence center view for the target area is recommended for better results. You can see this scenario, top-right of Figure 14, where the model wrongly classifies the field area present in the image as low income while it's not.

By combining localization, Figure 14 and mapping, Figure 13 with coordinates, the results obtained in this research could be used to monitor and enforce the implementation of government proposed zoning plans.

\section{CONCLUSION}

Monitoring and assessing the distribution of economic areas in East Africa such as low and high income neighborhoods, has typically relied on the use of structured data and traditional survey approaches for collecting information such as questionnaires, interviews and field visits. These types of surveys are often slow, costly and prone to human error. By using Deep Learning methods and satellite imagery, in this research study we achieve $98 \%$ accuracy results with our best model on the classification of five economic area categories. namely, Commercial, Industrial, Residential High, Residential Middle, and Residential Low. The best performing model in terms of an accuracy of $98 \%$ was Resnet50V2. The results show that given a specific land zone, one could assess with confidence the spatial distribution of various economic areas in that zone. This has applications in city infrastructure planning and policy monitoring such as city infrastructure master plan enforcement, city expansion monitoring and shanty towns formulation localization and prevention. Classification results express that the deep learning models used were able to learn different patterns independently by observing the ranking accuracy per category. Additional studies of blended optimal deep model architectures for remote sensing would be interesting to explore and most likely yield improved results across the categories.

\section{REFERENCES}

[1] Facundo Alvaredo et al. World inequality report 2018. Belknap Press, 2018.

[2] Gideon Baffoe et al. "Understanding the concept of neighbourhood in Kigali City, Rwanda". In: Sustainability 12.4 (2020), p. 1555.
[3] Jerzy Bański and Marcin Mazur. "Classification of rural areas in Poland as an instrument of territorial policy". In: Land Use Policy 54 (2016), pp. 1-17.

[4] Javier Barbero, Kristian Behrens, and Jose L Zofio. "Industry location and wages: The role of market size and accessibility in trading networks". In: Regional Science and Urban Economics 71 (2018), pp. 1-24.

[5] et al Barton Michael S. "An investigation of the influence of the spatial distribution of neighborhood violent crime on fear of crime". In: Crime Delinquency (2017), pp. 1757-1776.

[6] Sabyasachi Basu and Thomas G. Thibodeau. "Analysis of spatial autocorrelation in house prices". In: The Journal of Real Estate Finance and Economics (1998), pp. 61-85.

[7] Ricardo N Bebczuk and Ricardo Néstor Bebczuk. Asymmetric information in financial markets: introduction and applications. Cambridge University Press, 2003.

[8] Rodrigo F Berriel et al. "Deep learning-based largescale automatic satellite crosswalk classification". In: IEEE Geoscience and Remote Sensing Letters 14.9 (2017), pp. 1513-1517.

[9] Benjamin Bischke et al. "Multi-task learning for segmentation of building footprints with deep neural networks". In: 2019 IEEE International Conference on Image Processing (ICIP). IEEE. 2019, pp. 1480-1484.

[10] Desamparados Blazquez and Josep Domenech. "Big Data sources and methods for social and economic analyses". In: Technological Forecasting and Social Change 130 (2018), pp. 99-113.

[11] Kenneth K Boyer, John R Olson, and Eric C Jackson. "Electronic surveys: Advantages and disadvantages over traditional print surveys". In: Decision Line 32.4 (2001), pp. 4-7.

[12] Kimberly A. Clausing. "Does tax drive the headquarters locations of the world's biggest companies?" In: Transnational Corporations Journal (2018).

[13] Barney Cohen. "Urbanization in developing countries: Current trends, future projections, and key challenges for sustainability". In: Technology in society 28.1-2 (2006), pp. 63-80.

[14] Konrad Czapiewski, Jerzy Bański, and Magdalena Górczyńska. "The impact of location on the role of small towns in regional development: Mazovia, Poland". In: European Countryside 8.4 (2016), pp. 413-426. 


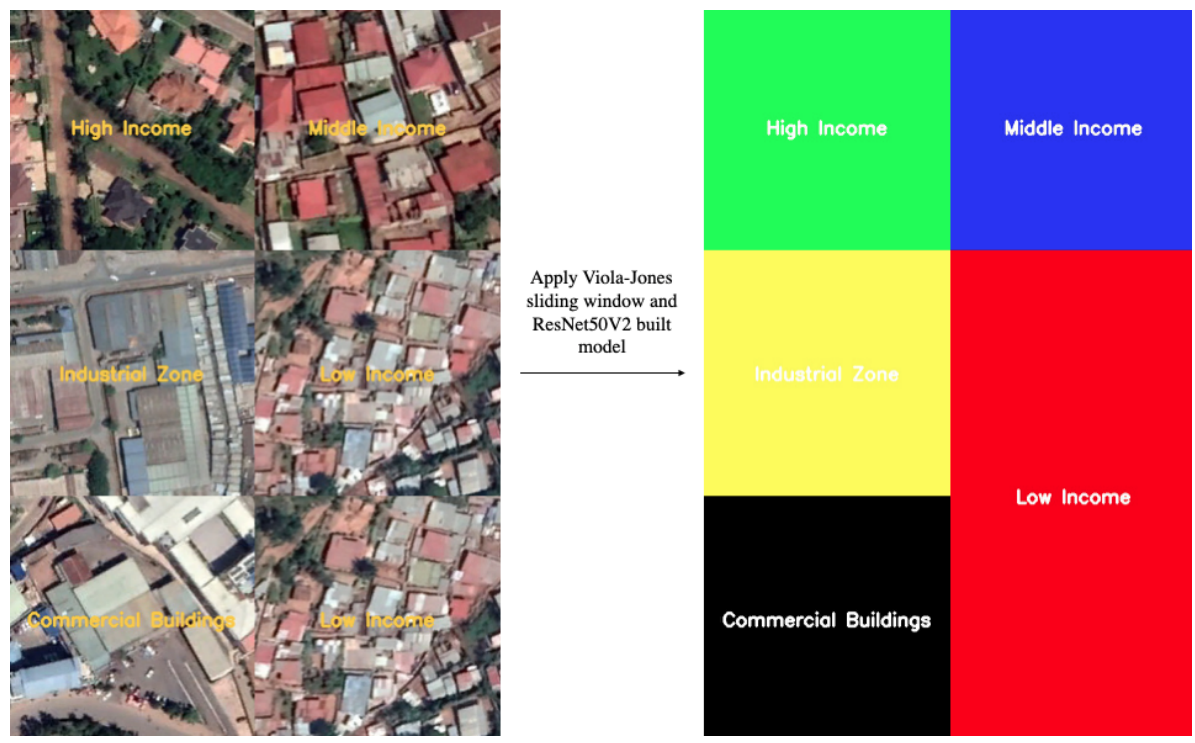

FIGURE 13. Input and output regions for instant mapping and legend using Resnet50V2.

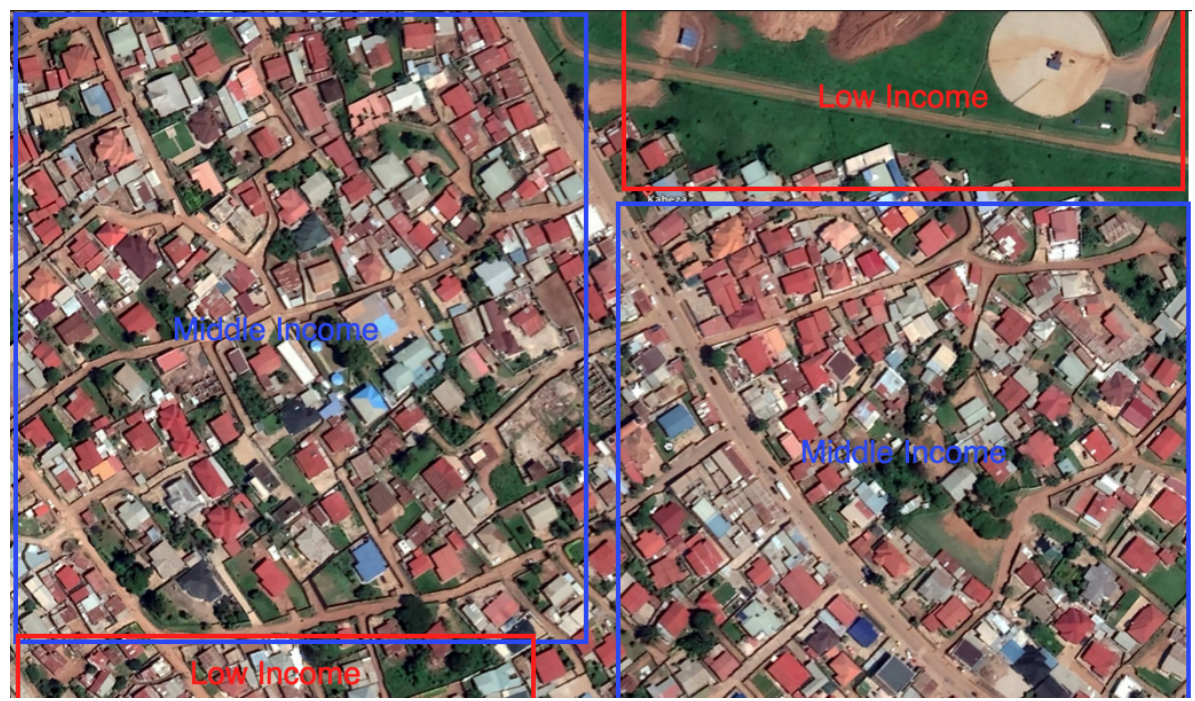

FIGURE 14. Middle income region ResNet50V2 detection results.

[15] James Daniell, Friedemann Wenzel, and Andreas Schaefer. "The economic costs of natural disasters globally from 1900-2015: historical and normalised floods, storms, earthquakes, volcanoes, bushfires, drought and other disasters". In: EGUGA (2016), EPSC2016-1899.

[16] Gary K Davis. "History of the NOAA satellite program". In: Journal of Applied Remote Sensing 1.1 (2007), p. 012504.

[17] Annette J Dobson and Adrian G Barnett. An introduction to generalized linear models. CRC press, 2018.

[18] Antonino Furnari et al. "Distortion adaptive Sobel filters for the gradient estimation of wide angle images".
In: Journal of Visual Communication and Image Representation 46 (2017), pp. 165-175.

[19] Yanming Guo et al. "Deep learning for visual understanding: A review". In: Neurocomputing 187 (2016), pp. 27-48.

[20] et al. He Zhanjun. "Discovering the joint influence of urban facilities on crime occurrence using spatial colocation pattern mining". In: Cities 99 (2020).

[21] Kaiming He et al. "Deep residual learning for image recognition". In: Proceedings of the IEEE conference on computer vision and pattern recognition. 2016, pp. $770-778$. 
[22] Kaiming He et al. "Identity mappings in deep residual networks". In: European conference on computer vision. Springer. 2016, pp. 630-645.

[23] Charles Hodgson. "The effect of transport infrastructure on the location of economic activity: Railroads and post offices in the American West". In: Journal of Urban Economics 104 (2018), pp. 59-76.

[24] Akanksha Kapoor and Abhishek Singhal. "A comparative study of K-Means, K-Means++ and Fuzzy C-Means clustering algorithms". In: 2017 3rd international conference on computational intelligence \& communication technology (CICT). IEEE. 2017, pp. $1-6$.

[25] Muhammad Jaleed Khan et al. "Automatic target detection in satellite images using deep learning". In: $J$. Space Technol 7.1 (2017), pp. 44-49.

[26] Nataliia Kussul et al. "Deep learning classification of land cover and crop types using remote sensing data". In: IEEE Geoscience and Remote Sensing Letters 14.5 (2017), pp. 778-782.

[27] Demetris Lamnisos et al. "Geodemographic Area Classification and Association with Mortality: An Ecological Study of Small Areas of Cyprus". In: International journal of environmental research and public health 16.16 (2019), p. 2927.

[28] Jong-Wha Lee and Innwon Park. "Free Trade Areas in East Asia: Discriminatory or Non-discriminatory?" In: World Economy 28.1 (2005), pp. 21-48.

[29] Lin Li, Yohei Sato, and Haihong Zhu. "Simulating spatial urban expansion based on a physical process". In: Landscape and urban planning 64.1-2 (2003), pp. 67-76.

[30] Minghao Li et al. Location Determinants of high Growth Firms. MPRA Paper. University Library of Munich, Germany, 2015. URL: https ://EconPapers . repec.org/RePEc:pra:mprapa:66611.

[31] Xi Li, Fengrui Chen, and Xiaoling Chen. "Satelliteobserved nighttime light variation as evidence for global armed conflicts". In: IEEE Journal of Selected Topics in Applied Earth Observations and Remote Sensing 6.5 (2013), pp. 2302-2315.

[32] KJ Linthicum et al. "Application of polar-orbiting, meteorological satellite data to detect flooding of Rift Valley Fever virus vector mosquito habitats in Kenya". In: Medical and Veterinary Entomology 4.4 (1990), pp. 433-438.

[33] Yanchi Liu et al. "Intelligent bus routing with heterogeneous human mobility patterns". In: Knowledge and Information Systems 50.2 (2017), pp. 383-415.

[34] Héctor A López-Ospina, Francisco J Martınez, and Cristián E Cortés. "Microeconomic model of residential location incorporating life cycle and social expectations". In: Computers, Environment and Urban Systems 55 (2016), pp. 33-43.
[35] Shuaib Lwasa and Cecilia Kinuthia-Njenga. "Reappraising urban planning and urban sustainability in East Africa". In: Urban development (2012), pp. 3-21.

[36] Yueliang Ma and Ruisong Xu. "Remote sensing monitoring and driving force analysis of urban expansion in Guangzhou City, China”. In: Habitat International 34.2 (2010), pp. 228-235.

[37] Laurens van der Maaten and Geoffrey Hinton. "Visualizing data using t-SNE". In: Journal of machine learning research 9.Nov (2008), pp. 2579-2605.

[38] Danny MacKinnon, Gordon Pirie, and Matthias Gather. "Transport and economic development". In: (2008).

[39] Rahel Meili and Heike Mayer. "Small and mediumsized towns in Switzerland: Economic heterogeneity, socioeconomic performance and linkages". In: Erdkunde (2017), pp. 313-332.

[40] Charlotta Mellander et al. "Night-time light data: A good proxy measure for economic activity?" In: PloS one 10.10 (2015), e0139779.

[41] Harvey J Miller and Yi-Hwa Wu. "GIS software for measuring space-time accessibility in transportation planning and analysis". In: GeoInformatica 4.2 (2000), pp. 141-159.

[42] Barak Oshri et al. "Infrastructure quality assessment in africa using satellite imagery and deep learning". In: Proceedings of the 24th ACM SIGKDD International Conference on Knowledge Discovery \& Data Mining. 2018, pp. 616-625.

[43] Sinno Jialin Pan and Qiang Yang. "A survey on transfer learning". In: IEEE Transactions on knowledge and data engineering 22.10 (2009), pp. 1345-1359.

[44] Andrew V Papachristos and Sara Bastomski. "Connected in crime: the enduring effect of neighborhood networks on the spatial patterning of violence". In: American Journal of Sociology 124.2 (2018), pp. 517568.

[45] Kirsteen Paton. "Making working-class neighbourhoods posh? Exploring the effects of gentrification strategies on working-class communities". In: Classed intersections: Spaces, selves, knowledges (2010), pp. 137-157.

[46] Matti Pietikäinen et al. Computer vision using local binary patterns. Vol. 40. Springer Science \& Business Media, 2011.

[47] Debraj Roy and Michael Lees. "Understanding resilience in slums using an agent-based model". In: Computers, Environment and Urban Systems 80 (2020), p. 101458.

[48] Jason Russ. "Essays on the Impact of Weather on Economic Activity". PhD thesis. The George Washington University, 2018.

[49] Mark Sandler et al. "Mobilenetv2: Inverted residuals and linear bottlenecks". In: Proceedings of the IEEE conference on computer vision and pattern recognition. 2018, pp. 4510-4520. 
[50] Alain Segessemann and Olivier Crevoisier. "Beyond economic base theory: The role of the residential economy in attracting income to Swiss regions". In: Regional Studies 50.8 (2016), pp. 1388-1403.

[51] Ling Shao, Fan Zhu, and Xuelong Li. "Transfer learning for visual categorization: A survey". In: IEEE transactions on neural networks and learning systems 26.5 (2014), pp. 1019-1034.

[52] Paheding Sidike et al. "dPEN: deep Progressively Expanded Network for mapping heterogeneous agricultural landscape using WorldView-3 satellite imagery”. In: Remote sensing of environment 221 (2019), pp. 756-772.

[53] Timothy F Slaper, Karter M Harmon, and Barry M Rubin. "Industry clusters and regional economic performance: A study across US metropolitan statistical areas". In: Economic Development Quarterly 32.1 (2018), pp. 44-59.

[54] Diana Suhardiman, Oulavanh Keovilignavong, and Miles Kenney-Lazar. "The territorial politics of land use planning in Laos". In: Land use policy 83 (2019), pp. 346-356.

[55] Christian Szegedy et al. "Rethinking the inception architecture for computer vision". In: Proceedings of the IEEE conference on computer vision and pattern recognition. 2016, pp. 2818-2826.

[56] Chuanqi Tan et al. "A survey on deep transfer learning”. In: International conference on artificial neural networks. Springer. 2018, pp. 270-279.

[57] JD Tarpley, SR Schneider, and RL Money. "Global vegetation indices from the NOAA-7 meteorological satellite". In: Journal of Climate and Applied Meteorology 23.3 (1984), pp. 491-494.

[58] Grigorios Tsagkatakis et al. "Survey of deep-learning approaches for remote sensing observation enhancement”. In: Sensors 19.18 (2019), p. 3929.

[59] Ernest Uwayezu and Walter T de Vries. "Access to Affordable Houses for the Low-Income Urban Dwellers in Kigali: Analysis Based on Sale Prices”. In: Land 9.3 (2020), p. 85.

[60] Adam Van Etten. "You only look twice: Rapid multiscale object detection in satellite imagery". In: arXiv preprint arXiv:1805.09512 (2018).

[61] Paul Viola and Michael Jones. "Rapid object detection using a boosted cascade of simple features". In: Proceedings of the 2001 IEEE computer society conference on computer vision and pattern recognition. CVPR 2001. Vol. 1. IEEE. 2001, pp. I-I.

[62] Athanasios Voulodimos et al. "Deep learning for computer vision: A brief review". In: Computational intelligence and neuroscience 2018 (2018).

[63] Ryan W Wolcott and Ryan M Eustice. "Visual localization within lidar maps for automated urban driving”. In: 2014 IEEE/RSJ International Conference on Intelligent Robots and Systems. IEEE. 2014, pp. 176183.
[64] James Yoo and Richard Ready. "The impact of agricultural conservation easement on nearby house prices: Incorporating spatial autocorrelation and spatial heterogeneity". In: Journal of Forest Economics (2016), pp. 78-93. 